\title{
Effect of initial antifungal therapy on mortality among patients with bloodstream infections with different Candida species and resistance to antifungal agents: A multicentre observational study by the Turkish Fungal Infections Study Group
}

\author{
Özlem Doğan a , Ayşegül Yeşilkaya ${ }^{\mathrm{b}}$, Şirin Menekşe ${ }^{c}$, Özlem Güler ${ }^{\mathrm{d}}$, Çağla Karakoç ${ }^{\mathrm{e}}$, \\ Güle Çınarf, Mahir Kapmaz ${ }^{\mathrm{a}}$, Mehtap Aydın' ${ }^{\mathrm{g}}$, Şiran Keske ${ }^{\mathrm{h}}$, Suzan Şahin ${ }^{\mathrm{i}}$, \\ Demet Hacıseyitoğlu ${ }^{i}$, Demet Yalçın ${ }^{j}$, Süda Tekin ${ }^{a}$, Nazlı Ataça ${ }^{a}$ Özgür Albayrak ${ }^{a}$, \\ Ekin Deniz Aksua ${ }^{a}$, Füsun Can ${ }^{a}$, Önder Ergönül ${ }^{\mathrm{a}, *}$ \\ a Department of Infectious Diseases and Clinical Microbiology, Koç University, Istanbul, Turkey \\ ${ }^{\mathrm{b}}$ Department of Infectious Diseases and Clinical Microbiology, Başkent University, Ankara, Turkey \\ ${ }^{c}$ Department of Infectious Diseases and Clinical Microbiology, Koşuyolu State Hospital, Istanbul, Turkey \\ ${ }^{\mathrm{d}}$ Department of Infectious Diseases and Clinical Microbiology, Kocaeli University, Kocaeli, Turkey \\ e Department of Infectious Diseases and Clinical Microbiology, Liv Hospital, Istanbul, Turkey \\ ${ }^{\mathrm{f}}$ Department of Infectious Diseases and Clinical Microbiology, Ankara University, Istanbul, Turkey \\ g Department of Infectious Diseases and Clinical Microbiology, University of Health Sciences, Istanbul, Turkey

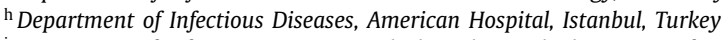 \\ i Department of Infectious Diseases and Clinical Microbiology, Dr Lütfü Kirdar Research and Training Hospital, Istanbul, Turkey \\ j Department of Infectious Diseases and Clinical Microbiology, Göztepe Medicalpark, Istanbul, Turkey
}

\section{A R T I C L E I N F O}

\section{Article history:}

Received 21 January 2020

Accepted 15 April 2020

Available online $\mathrm{xxx}$

Editor: Dr. Stephane Ranque

\section{Keywords:}

Candidaemia

Echinocandins

Fluconazole

Azole resistance

Antifungal susceptibility

Clinical impact

\begin{abstract}
A B S T R A C T
This study aimed to describe the effect of initial antifungal therapy on patient mortality and to detail the current distribution and resistance patterns of Candida spp. among patients with candidaemia. A prospective observational study was performed among consecutive patients with candidaemia from 10 Turkish medical centres between January 2015 and November 2018. The primary outcome was 10-day mortality. Species were identified using MALDI-TOF/MS. A total of 342 patients with candidaemia were included, of which $175(51.2 \%)$ were male and $68(19.9 \%)$ were aged $<18$ years. The most common species were Candida albicans (47.4\%), Candida parapsilosis (26.6\%), Candida tropicalis (9.6\%) and Candida glabrata (7.6\%). Among all Candida spp., the 10-day case fatality rate (CFR) was $32.2 \%$. The CFR was highest in patients with C. albicans (57.3\%) and lowest in patients with C. parapsilosis (21.8\%). The resistance rate to fluconazole was $13 \%$ in C. parapsilosis, with no significant effect on mortality. No resistance to echinocandins was detected. In the multivariate analysis, being in the ICU [OR $=2.1(95 \% \mathrm{CI} 1.32-3.57) ; P=0.002$ ], renal failure [OR $=2.4$ (1.41-3.97); $P=0.001$ ], total parenteral nutrition [OR $=2(1.22-3.47) ; P=0.006], C$. albicans infection [OR $=1.7(1.06-2.82) ; P=0.027$ ] and echinocandin as primary agent [OR $=0.6(0.36-$ 0.99 ); $P=0.047$ ] were significantly associated with mortality. Candidaemia is a deadly infection. Fluconazole resistance is emerging, although it was not significantly related to mortality. Using an echinocandin as the primary agent could be life-saving.
\end{abstract}

(c) 2020 Elsevier B.V. and International Society of Chemotherapy. All rights reserved.

\section{Introduction}

Candida spp. are the fourth and seventh most common cause of healthcare-associated bloodstream infections (BSIs) in the USA

\footnotetext{
* Corresponding author.

E-mail address: oergonul@ku.edu.tr (Ö. Ergönül).
}

and Europe, respectively [1,2]. According to hospital-based studies, the global incidence of candidaemia varies from 0.3 to 5 per 1000 admissions [3]. The attributable mortality rate of candidaemia was reported as between $5-71 \%$ [4]. In critically ill patients, both patient co-morbidities and the infection itself contribute to mortality [5]. The risk of mortality can be reduced by early initiation of appropriate antifungal treatment [6]. It is known that one of the 
main risks for increased mortality in candidaemia is a delay in appropriate antifungal treatment until positive blood culture [7]. Despite the known risk factors, candidaemia remains associated not only with a high mortality rate but also prolonged hospital stays and increased hospital costs [8].

The distribution of Candida spp. causing candidaemia varies according to geographic location, patient population and antifungal stewardship policies. Candida albicans remains the most frequently isolated species in almost every centre globally, however some studies report an increasing rate of isolation of non-albicans Candida spp. [9]. In a single-centre study from France, the change in species distribution of candidaemia isolates over a decade was evaluated and no significant differences was found between 2000 and 2010 [10]. In the USA and Northern Europe, Candida glabrata is reported as the second most common causative agent of candidaemia, whereas Candida parapsilosis is the most commonly isolated species following C. albicans in Southern Europe and Latin America [11]. A study by Ding et al. found previous azole therapy [odds ratio $(\mathrm{OR})=3.359,95 \%$ confidence interval (CI) 1.136-10.154; $P=0.031$ ] and use of artificial surgical implants (OR $=37.519,95 \% \mathrm{CI} 2.5-562.998 ; P=0.009$ ) to be significant risk factors for infection with non-albicans Candida spp. [12]. In another multicentre study, the mortality rate was found to be significantly higher in infections by non-albicans Candida spp. than infections by $C$. albicans ( $65 \%$ vs. $53 \% ; P=0.10)$, and female sex $(O R=2.09$, 95\% CI 1.13-3.86) and increased duration of central venous catheter $(\mathrm{CVC})$ use ( $\mathrm{OR}=1.16$ per 5-day interval, 95\% CI 1.05-1.28) were found to be an independent risk factors for non-albicans candidaemia [13].

According to several guidelines, fluconazole or echinocandins may be considered for initial treatment of Candida BSI $[14,15]$. The severity of underlying diseases and co-morbidities of the patient, the epidemiological distribution of the causative agents, and the resistance rates can directly effect the choice of the primary drug for therapy of candidaemia. This study aimed to describe the effect of initial antifungal therapy on mortality among patients with candidaemia and to detail the current distribution and resistance patterns of Candida spp. isolates.

\section{Methods}

\subsection{Study design and population}

A prospective observational study was performed among consecutive patients with candidaemia from 10 Turkish medical centres between January 2015 and November 2018. The design of the study fulfilled the STROBE (Strengthening the Reporting of Observational Studies in Epidemiology) criteria. An episode of candidaemia was defined as the isolation of a Candida sp. isolate from blood cultures. Patient cultures with two or more fungal species were excluded from the analysis. The primary outcome was 10-day mortality. Treatment success was defined as clinical and mycological response at the end of therapy. Demographic and clinical data [age, sex, being in the intensive care unit (ICU), operation within previous month, antibiotic use within previous month, presence of malignancy, neutropenia, renal failure, solid-organ transplantation, presence of a CVC, total parenteral nutrition, usage of mechanical ventilation, prior antifungal exposure and initial antifungal therapy] were recorded on a standardised case report form. Initial antifungal therapy was defined as the primary antifungal treatment, either empirical or targeted, given to the patient for $\geq 96 \mathrm{~h}$. Patients receiving amphotericin $\mathrm{B}$ or voriconazole and patients who did not receive any treatment were excluded from the analysis of initial antifungal therapy.

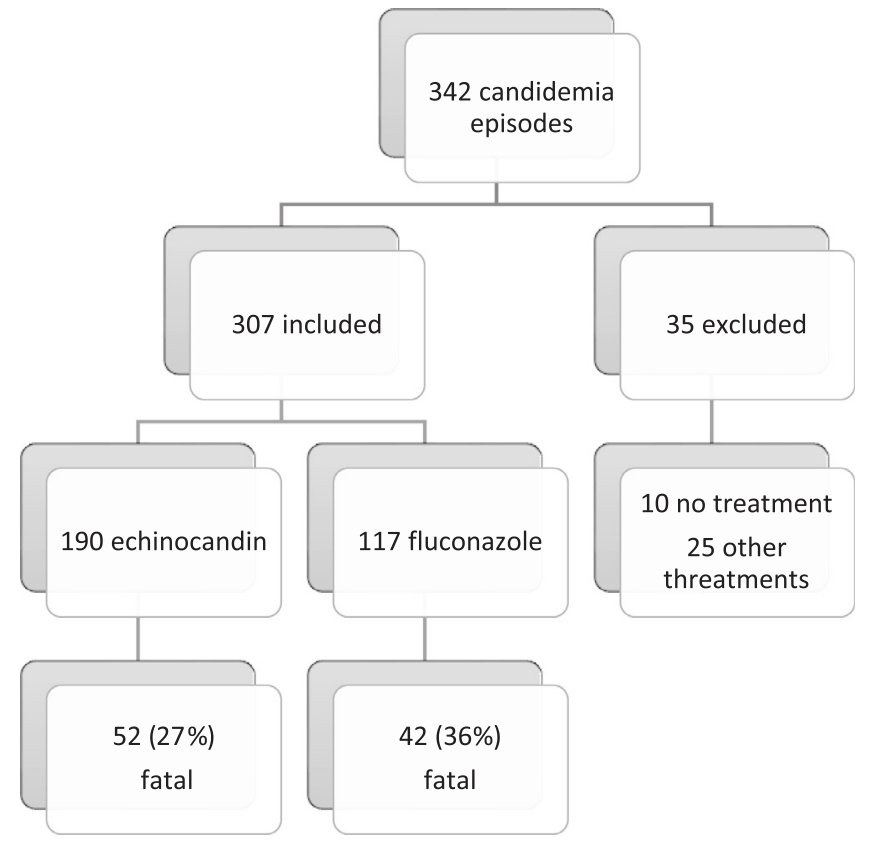

Fig. 1. Flowchart of candidaemia patients included in the study and initial antifungal therapy.

\subsection{Mycological studies}

For microbiological evaluation, all isolates were identified in the participating hospital laboratories and were confirmed by matrixassisted laser desorption/ionisation time-of-flight mass spectrometry (MALDI-TOF/MS) (bioMérieux, Lyon, France) in the reference mycology laboratory (Mycology Laboratory, Koç University Hospital, Istanbul, Turkey). Antifungal susceptibility testing for fluconazole, voriconazole, posaconazole, caspofungin and amphotericin B was performed by the broth microdilution method according to Clinical and Laboratory Standards Institute (CLSI) guidelines [16]. Minimum inhibitory concentrations (MICs) were evaluated following incubation for $24 \mathrm{~h}$ and $48 \mathrm{~h}$. For interpretation of antifungal susceptibility test results, if possible species-specific clinical breakpoints or epidemiological cut-off values were used as recommended by the CLSI $[17,18]$.

\subsection{Statistical data}

Data were analysed using Stata Statistical Software: Release 14.2 (StataCorp LP, College Station, TX, USA). Continuous variables were compared by Student's t-test or Mann-Whitney U-test. Categorical variables were compared by $\chi^{2}$ test. Statistical significance was determined using two-tailed tests, and a $P$-value of $<0.05$ was considered statistically significant. Variables that were found to have a significant effect on 10-day mortality in the univariate analysis were included in the multivariate model, and logistic regression was performed calculating the OR and corresponding 95\% CI.

\section{Results}

A total of 342 patients with candidaemia were enrolled in the study, among which 35 patients were excluded (10 did not receive any antifungal therapy, 23 received amphotericin $B$ and 2 received voriconazole). Among the 307 remaining patients, initial antifungal therapy was an echinocandin in 190 patients and fluconazole in 117 patients (Fig. 1).

Patients with $C$. parapsilosis were the youngest (mean age 42 years), whereas patients with $C$. glabrata infection were the old- 
Table 1

Predictors of 10-day mortality among patients with candidaemia in the univariate analysis ${ }^{\mathrm{a}}$.

\begin{tabular}{llll}
\hline Variable & Survived $(n=232)$ & Died $(n=110)$ & $P$-value \\
\hline Age (years) (mean \pm S.D.) & $48 \pm 27$ & $51 \pm 25$ & 0.212 \\
Female sex & $114(49.1)$ & $53(48.2)$ & 0.869 \\
Being in the ICU & $74(31.9)$ & $62(56.4)$ & $<0.001$ \\
Operation within previous month & $122(52.6)$ & $58(52.7)$ & 0.981 \\
Antibiotic use in previous month & $204(87.9)$ & $105(95.5)$ & 0.028 \\
Malignancy & $92(39.7)$ & $37(33.6)$ & 0.283 \\
Renal failure & $60(25.9)$ & $51(46.4)$ & $<0.001$ \\
Transplantation & $17(7.3)$ & $6(5.5)$ & 0.518 \\
CVC & $188(81.0)$ & $98(89.1)$ & 0.060 \\
Total parenteral nutrition & $127(54.7)$ & $80(72.7)$ & 0.001 \\
Mechanical ventilation & $42(18.1)$ & $19(17.3)$ & 0.851 \\
Candida albicans & $99(42.7)$ & $63(57.3)$ & 0.012 \\
Candida parapsilosis & $67(28.9)$ & $24(21.8)$ & 0.167 \\
Candida tropicalis & $26(11.2)$ & $7(6.4)$ & 0.156 \\
Candida glabrata & $21(9.1)$ & $5(4.5)$ & 0.142 \\
Echinocandin use as primary agent & $138(59.5)$ & $52(47.3)$ & 0.034 \\
Fluconazole use as primary agent & $75(32.3)$ & $42(38.2)$ & 0.286 \\
Fluconazole resistance & $9(3.9)$ & $3(2.7)$ & 0.323 \\
\hline
\end{tabular}

S.D., standard deviation; ICU, intensive care unit; CVC, central venous catheter

a Data are $n(\%)$ unless otherwise stated.

Table 2

Predictors of 10-day mortality among patients with candidaemia in the multivariate analysis.

\begin{tabular}{|c|c|c|c|c|c|c|}
\hline \multirow[t]{2}{*}{ Variable } & \multicolumn{3}{|c|}{ Unadjusted } & \multicolumn{3}{|c|}{ Adjusted } \\
\hline & OR & $95 \% \mathrm{CI}$ & $P$-value & OR & $95 \% \mathrm{CI}$ & $P$-value \\
\hline Being in the ICU & 2.7 & $1.72-4.39$ & $<0.001$ & 2.1 & $1.32-3.57$ & 0.002 \\
\hline Antibiotic use within previous month & 2.8 & $1.08-7.68$ & 0.034 & - & - & - \\
\hline Renal failure & 2.5 & $1.53-3.98$ & $<0.001$ & 2.4 & $1.41-3.97$ & 0.001 \\
\hline Total parenteral nutrition & 2.2 & $1.34-3.6$ & 0.002 & 2 & $1.22-3.47$ & 0.006 \\
\hline Candida albicans & 1.8 & $1.13-2.84$ & 0.012 & 1.7 & $1.06-2.82$ & 0.027 \\
\hline Echinocandin use as primary agent & 0.6 & $0.38-0.96$ & 0.034 & 0.6 & $0.36-0.99$ & 0.047 \\
\hline
\end{tabular}

OR, odds ratio; $\mathrm{CI}$, confidence interval; ICU, intensive care unit.

est (mean age 57 years). Among the 342 included patients, 175 $(51.2 \%)$ were male and $68(19.9 \%)$ were aged $<18$ years. Among all the patients, $28(8.2 \%)$ had previously received an antifungal drug (20 fluconazole, 5 echinocandin and 3 both fluconazole and an echinocandin). Among the 28 patients who had received previous antifungal treatment, 11 (39\%) had C. albicans infection and 17 (61\%) had non-albicans Candida infection. Among all Candida spp., the 10 -day case fatality rate (CFR) was $32.2 \%$. Independent predictors of mortality are shown in Table 1.

In the multivariate analysis, being in the ICU $(\mathrm{OR}=2.1$, 95\% CI 1.32-3.57; $P=0.002$ ), renal failure $(\mathrm{OR}=2.4,95 \% \mathrm{CI}$ 1.41-3.97; $P=0.001)$, total parenteral nutrition (OR $=2,95 \%$ CI 1.22-3.47; $P=0.006)$ and $C$. albicans infection $(\mathrm{OR}=1.7,95 \% \mathrm{CI}$ $1.06-2.82 ; P=0.027$ ) were found to be significantly positively associated with 10-day mortality. In contrast, using an echinocandin as the primary agent was significantly negatively associated with 10 -day mortality ( $\mathrm{OR}=0.6,95 \% \mathrm{CI} 0.36-0.99 ; P=0.047)$ (Table 2 ).

The most common Candida spp. were C. albicans (47.4\%), C. parapsilosis (26.6\%), Candida tropicalis (9.6\%) and C. glabrata (7.6\%). Species-specific patient characteristics are presented in Table 3. The highest CFR was among patients with C. albicans (57.3\%; $P=0.012$ ) and the lowest was among patients with $C$. parapsilosis (21.8\%; $P=0.167$ ). The resistance rate to fluconazole was $13 \%$ in C. parapsilosis isolates. Among 12 patients with fluconazoleresistant $C$. parapsilosis isolates, three infections were fatal. The choice of initial antifungal therapy was fluconazole for 5 of 12 fluconazole-resistant $C$. parapsilosis infections before the positive blood culture and antifungal susceptibility test results were obtained. Among these five patients, one patient died who had received fluconazole for the first 7 days and caspofungin for the next 6 days. Fluconazole resistance has no significant effect on mor- tality $(P=0.167)$ compared with fluconazole-susceptible $C$. parapsilosis infections. No resistance to echinocandins was detected (Table 4).

\section{Discussion}

In this multicentre prospective observational study, initial echinocandin therapy was found to be associated with a better outcome (OR $=0.6,95 \%$ CI $0.36-0.99 ; P=0.047$ ). Current guidelines from the Infectious Diseases Society of America (IDSA) recommend an echinocandin as the first-line therapy of candidaemia in clinically moderate and severe patients [14]. In a patient-level quantitative review of seven randomised trials, echinocandins were found to be associated with better survival rates than either triazoles or polyenes [19]. To date, there is only one randomised controlled study comparing fluconazole with an echinocandin for the treatment of Candida BSI, in which fluconazole was associated with lower success rates compared with anidulafungin [20]. In a recent large retrospective cohort analysis, initial echinocandin treatment was found to be associated with decreased hospital mortality compared with fluconazole $(\mathrm{OR}=0.22,95 \%$ CI $0.06-0.85 ; P=0.028)$ [21].

On the other hand, some studies obtained discordant results regarding the superiority of empirical echinocandin treatment among patients with candidaemia. In a multicentre prospective cohort study from Spain, López-Cortés et al. found that empirical therapy with fluconazole was associated with a better prognosis (adjusted hazard ratio $=0.38,95 \% \mathrm{Cl} 0.17-0.81 ; P=0.01$ ) but this association disappeared in the propensity score-based stratified and matched analyses, which could be the result of the control of confounding variables [22]. In another multicentre cohort study, no difference 
Table 3

Species-specific characteristics of patients with candidaemia ${ }^{\mathrm{a}}$.

\begin{tabular}{|c|c|c|c|c|c|c|}
\hline \multirow[t]{2}{*}{ Characteristic } & \multirow{2}{*}{$\begin{array}{l}\text { C. albicans } \\
(n=162)\end{array}$} & \multicolumn{5}{|c|}{ Non-albicans Candida spp. } \\
\hline & & Total $(n=180)$ & C. parapsilosis $(n=91)$ & C. tropicalis $(n=33)$ & C. glabrata $(n=26)$ & Others $^{\mathrm{b}}(n=30)$ \\
\hline 10-day mortality & $63(38.9)$ & $47(26.1)$ & $24(26)$ & $7(21)$ & $5(19)$ & $11(37)$ \\
\hline Age (years) (mean \pm S.D.) & $51.4 \pm 26$ & $46.4 \pm 26$ & $41.5 \pm 28$ & $46.2 \pm 26$ & $57.3 \pm 21$ & $52 \pm 26$ \\
\hline Female sex & $85(52.5)$ & $82(45.6)$ & $35(38)$ & $14(42)$ & $17(65)$ & $16(53)$ \\
\hline Being in the ICU & $67(41.4)$ & $69(38.3)$ & $48(53)^{*}$ & $10(30)$ & $4(15)$ & $7(23)$ \\
\hline Operation within previous month & $90(55.6)$ & $90(50.0)$ & $46(51)$ & $18(55)$ & $14(54)$ & $12(40)$ \\
\hline Antibiotic use within previous month & $146(90.1)$ & $163(90.6)$ & $84(92)$ & $31(94)$ & $22(85)$ & $26(87)$ \\
\hline Malignancy & $65(40.1)$ & $64(35.6)$ & $21(23)$ & $16(48)$ & $16(62)^{* *}$ & $11(37)$ \\
\hline Renal failure & $56(34.6)$ & $55(30.6)$ & $33(36)$ & $8(24)$ & $4(15)$ & $10(33)$ \\
\hline Transplantation & $8(4.9)$ & $15(8.3)$ & $5(5)$ & $2(6)$ & $2(8)$ & $6(20)$ \\
\hline CVC & $134(82.7)$ & $152(84.4)$ & $78(86)$ & $30(91)$ & $21(81)$ & $23(77)$ \\
\hline Total parenteral nutrition & $99(61.1)$ & $108(60.0)$ & $57(63)$ & $17(52)$ & $19(73)$ & $15(50)$ \\
\hline Mechanical ventilation & $35(21.6)$ & $26(14.4)$ & $18(20)$ & $4(12)$ & $1(4)$ & $3(10)$ \\
\hline Diabetes mellitus & $18(11.1)$ & $26(14.4)$ & $11(12)$ & $3(9)$ & $6(23)$ & $6(20)$ \\
\hline Echinocandin use as primary agent & $86(53.1)$ & $104(57.8)$ & $45(49)$ & $21(64)$ & $17(65)$ & $21(70)$ \\
\hline Fluconazole use as primary agent & $58(35.8)$ & $59(32.8)$ & $38(42)$ & $8(24)$ & $7(27)$ & $6(20)$ \\
\hline
\end{tabular}

S.D., standard deviation; ICU, intensive care unit; CVC, central venous catheter.

a Data are $n(\%)$ unless otherwise stated.

b C. krusei $(n=9)$, C. kefyr $(n=9)$, C. lusitaniae $(n=5)$, C. dubliniensis $(n=4)$ and C. guilliermondii $(n=3)$.

* $P=0.008$

** $P=0.009$.

Table 4

Minimum inhibitory concentration (MIC) distribution of the isolates.

\begin{tabular}{|c|c|c|c|c|c|}
\hline \multirow{2}{*}{$\begin{array}{l}\text { Species/antifungal } \\
\text { agent }\end{array}$} & \multicolumn{3}{|c|}{ MIC (mg/L) } & \multicolumn{2}{|c|}{ Susceptibility [n(\%)] } \\
\hline & $\mathrm{MIC}_{50}$ & $\mathrm{MIC}_{90}$ & Range & Resistant & Intermediate \\
\hline \multicolumn{6}{|c|}{ Candida albicans $(n=162)$} \\
\hline Fluconazole & 0.125 & 0.125 & $0.125-0.5$ & 0 & \\
\hline Voriconazole & 0.015 & 0.03 & $0.015-0.03$ & 0 & \\
\hline Posaconazole & 0.03 & 0.03 & $0.03-0.06$ & 0 & \\
\hline Caspofungin & 0.06 & 0.25 & $0.03-0.25$ & 0 & \\
\hline Amphotericin B & 1 & 1 & $0.5-1$ & 0 & \\
\hline \multicolumn{6}{|c|}{ Candida parapsilosis $(n=91)$} \\
\hline Fluconazole & 1 & 8 & $0.125-32$ & $12(13)$ & $8(9)$ \\
\hline Voriconazole & 0.015 & 0.5 & $0.015-0.5$ & 0 & $9(10)$ \\
\hline Posaconazole & 0.03 & 0.125 & $0.03-0.5$ & 0 & \\
\hline Caspofungin & 0.5 & 2 & $0.03-2$ & 0 & \\
\hline Amphotericin B & 1 & 2 & $0.5-2$ & 0 & \\
\hline \multicolumn{6}{|c|}{ Candida glabrata $(n=26)$} \\
\hline Fluconazole & 2 & 8 & $0.5-8$ & 0 & \\
\hline Voriconazole & 0.125 & 0.25 & $0.015-0.25$ & 0 & \\
\hline Posaconazole & 0.5 & 0.5 & $0.03-1$ & 0 & \\
\hline Caspofungin & 0.06 & 0.125 & $0.03-0.125$ & 0 & \\
\hline Amphotericin B & 2 & 2 & $1-2$ & 0 & \\
\hline \multicolumn{6}{|c|}{ Candida tropicalis $(n=33)$} \\
\hline Fluconazole & 0.5 & 1 & $0.125-2$ & 0 & \\
\hline Voriconazole & 0.03 & 0.06 & $0.015-0.125$ & 0 & \\
\hline Posaconazole & 0.03 & 0.06 & $0.03-0.125$ & 0 & \\
\hline Caspofungin & 0.125 & 0.25 & $0.03-0.25$ & 0 & \\
\hline Amphotericin B & 1 & 2 & $0.5-2$ & 0 & \\
\hline
\end{tabular}

$\mathrm{MIC}_{50 / 90}$, MICs for $50 \%$ and $90 \%$ of the isolates, respectively.

in mortality between two drugs was found after adjustment for Acute Physiology and Chronic Health Evaluation (APACHE) II scores in patients with septic shock due to candidaemia [23].

The overall 10-day CFR in this study was $32.2 \%$ and was highest (57.3\%; $P=0.012$ ) among patients with $C$. albicans BSI (OR $=1.7$, 95\% CI 1.06-2.82; $P=0.027)$. Candida parapsilosis was the second most commonly isolated species, similar to previous reports from Turkey and Southern Europe [11,24]. In the current study, 12 (13\%) of $91 \mathrm{C}$. parapsilosis isolates were found to be non-susceptible to fluconazole, which were obtained from patients in three different hospitals In a multicentre study from Turkey, the fluconazole resistance rate in C. parapsilosis was found be 7.7\% [25]. In a recent global antifungal surveillance analysis, among 225 C. parapsilosis isolates from Europe, the fluconazole resistance rate was $15.1 \%$ and the majority of the non-susceptible isolates were obtained from three hospitals in Italy and harbouring the same resistance mechanism [26]. In the current study, high resistance to fluconazole in C. parapsilosis isolates was detected with no significant effect on patient outcomes $(P=0.167)$. Despite the higher MICs of echinocandins against $C$. parapsilosis, initial echinocandin treatment was not associated with a negative outcome in $C$. parapsilosis BSI [27]. These results could be explained by the low virulence of $C$. parapsilosis, but further investigations should be performed on the resistance mechanisms and clonal distribution of the $C$. parapsilosis isolates.

Strengths of this study were being a large prospective clinical cohort including Candida BSI. All of the samples were re-tested in the reference mycology laboratory using MALDI-TOF/MS. The ref- 
erence broth microdilution method was used for antifungal susceptibility testing. A limitation of the study was the lack of disease severity scores for the patients, but it is more likely to give echinocandins for severe patients and, despite this fact, patients who received echinocandins as initial therapy had better outcomes in the multivariate analysis.

In conclusion, candidaemia is one of the most fatal infections. Resistance to fluconazole is emerging, although in this study it was not significantly related to mortality. Using echinocandins as the primary agent was found to be beneficial.

Funding: None.

Competing interests: None declared.

Ethical approval: This study was approved by the Institutional Review Board of Koç University (Istanbul, Turkey) [reference no. 2018.165.IRB2.029].

\section{References}

[1] Edmond MB, Wallace SE, McClish DK, Pfaller MA, Jones RN, Wenzel RP. Nosocomial bloodstream infections in United States hospitals: a three-year analysis. Clin Infect Dis 1999;29:239-44.

[2] Marchetti O, Bille J, Fluckiger U, Eggimann P, Ruef C, Garbino J, et al. Epidemiology of candidemia in Swiss tertiary care hospitals: secular trends, 1991-2000. Clin Infect Dis 2004;38:311-20.

[3] Falagas ME, Roussos N, Vardakas KZ. Relative frequency of albicans and the various non-albicans Candida spp among candidemia isolates from inpatients in various parts of the world: a systematic review. Int J Infect Dis 2010;14:e954-66.

[4] Falagas ME, Apostolou KE, Pappas VD. Attributable mortality of candidemia: a systematic review of matched cohort and case-control studies. Eur J Clin Microbiol Infect Dis 2006;25:419-25.

[5] Wisplinghoff $H$, Bischoff $T$, Tallent SM, Seifert H, Wenzel RP, Edmond MB. Nosocomial bloodstream infections in US hospitals: analysis of 24,179 cases from a prospective nationwide surveillance study. Clin Infect Dis 2004;39:309-17.

[6] Blot SI, Vandewoude KH, Hoste EA, Colardyn FA. Effects of nosocomial candidemia on outcomes of critically ill patients. Am J Med 2002;113:480-5.

[7] Morrell M, Fraser VJ, Kollef MH. Delaying the empiric treatment of Candida bloodstream infection until positive blood culture results are obtained: a potential risk factor for hospital mortality. Antimicrob Agents Chemother 2005;49:3640-5.

[8] Morgan J, Meltzer MI, Plikaytis BD, Sofair AN, Huie-White S, Wilcox S, et al. Excess mortality, hospital stay, and cost due to candidemia: a case-control study using data from population-based candidemia surveillance. Infect Control Hosp Epidemiol 2005;26:540-7.

[9] Taei M, Chadeganipour M, Mohammadi R. An alarming rise of non-albicans Candida species and uncommon yeasts in the clinical samples; a combination of various molecular techniques for identification of etiologic agents. BMC Res Notes 2019;12:779.

[10] Parmeland L, Gazon M, Guerin C, Argaud L, Lehot J-J, Bastien O, et al. Candida albicans and non-Candida albicans fungemia in an institutional hospital during a decade. Med Mycol 2013;51:33-7.

[11] Guinea J. Global trends in the distribution of Candida species causing candidemia. Clin Microbiol Infect 2014;20(Suppl 6):5-10.
[12] Ding X, Yan D, Sun W, Zeng Z, Su R, Su J. Epidemiology and risk factors for nosocomial non-Candida albicans candidemia in adult patients at a tertiary care hospital in North China. Med Mycol 2015:53:684-90.

[13] Holley A, Dulhunty J, Blot S, Lipman J, Lobo S, Dancer C, et al. Temporal trends, risk factors and outcomes in albicans and non-albicans candidaemia: an international epidemiological study in four multidisciplinary intensive care units. Int J Antimicrob Agents 2009;33:554.e1-554.e7.

[14] Pappas PG, Kauffman CA, Andes DR, Clancy CJ, Marr KA, Ostrosky-Zeichner L, et al. Clinical practice guideline for the management of candidiasis: 2016 update by the Infectious Diseases Society of America. Clin Infect Dis 2016;62:e1-50.

[15] Cornely OA, Bassetti M, Calandra T, Garbino J, Kullberg BJ, Lortholary O, et al. ESCMID* guideline for the diagnosis and management of Candida diseases 2012: non-neutropenic adult patients. Clin Microbiol Infect 2012;18(Suppl 7):19-37.

[16] Clinical and Laboratory Standards Institute (CLSI). Reference method for broth dilution antifungal susceptibility testing of yeasts; approved standard-third edition, Wayne, PA: CLSI; 2008. CLSI document M27-A3.

[17] Pfaller MA, Diekema DJ. Progress in antifungal susceptibility testing of Candida spp. by use of Clinical and Laboratory Standards Institute broth microdilution methods, 2010 to 2012. J Clin Microbiol 2012:50:2846-56.

[18] Clinical and Laboratory Standards Institute (CLSI). Reference method for broth dilution antifungal susceptibility testing of yeasts. 4th ed. Wayne, PA: CLSI; 2017. CLSI standard M27.

[19] Andes DR, Safdar N, Baddley JW, Playford G, Reboli AC, Rex JH, et al. Impact of treatment strategy on outcomes in patients with candidemia and other forms of invasive candidiasis: a patient-level quantitative review of randomized trials. Clin Infect Dis 2012:54:1110-22.

[20] Reboli AC, Rotstein C, Pappas PG, Chapman SW, Kett DH, Kumar D, et al. Anidulafungin versus fluconazole for invasive candidiasis. N Engl J Med 2007:356:2472-82

[21] Cui N, Wang H, Qiu H, Li R, Liu D. Impact of initial empirical antifungal agents on the outcome of critically ill patients with invasive candidiasis: analysis of the China-SCAN study. Int J Antimicrob Agents 2017;50:74-80.

[22] López-Cortés LE, Almirante B, Cuenca-Estrella M, Garnacho-Montero J, Padilla B, Puig-Asensio M, et al. Empirical and targeted therapy of candidemia with fluconazole versus echinocandins: a propensity score-derived analysis of a population-based, multicentre prospective cohort. Clin Microbiol Infect 2016:22:733.e1-733.e8.

[23] Bassetti M, Righi E, Ansaldi F, Merelli M, Trucchi C, De Pascale G, et al. A multicenter study of septic shock due to candidemia: outcomes and predictors of mortality. Intensive Care Med 2014:40:839-45.

[24] Alp S, Arikan-Akdagli S, Gulmez D, Ascioglu S, Uzun O, Akova M. Epidemiology of candidaemia in a tertiary care university hospital: 10-year experience with 381 candidaemia episodes between 2001 and 2010. Mycoses 2015:58:498-505.

[25] Arikan-Akdagli S, Gulmez D, Dogan O, Cerikcioglu N, Doluca Dereli M, Birinci $\mathrm{A}$, et al. First multicentre report of in vitro resistance rates in candidaemia isolates in Turkey. J Glob Antimicrob Resist 2019;18:230-4.

[26] Castanheira M, Deshpande LM, Messer SA, Rhomberg PR, Pfaller MA. Analysis of global antifungal surveillance results reveals predominance of Erg11 Y132F alteration among azole-resistant Candida parapsilosis and Candida tropicalis and country-specific isolate dissemination. Int I Antimicrob Agents 2020;55:105799.

[27] Fernandez-Ruiz M, Aguado JM, Almirante B, Lora-Pablos D, Padilla B, Puig-Asensio $\mathrm{M}$, et al. Initial use of echinocandins does not negatively influence outcome in Candida parapsilosis bloodstream infection: a propensity score analysis. Clin Infect Dis 2014;58:1413-21. 